\title{
A Rapid, Efficient and Green Method for Synthesis of 3,3'-Arylmethylene-bis-4-hydroxycoumarins without Use of any Solvent, Catalyst or Solid Surface
}

\author{
ARPITA DAS GUPTA, SWATI SAMANTA, RINA MONDAL and ASOK K. MALLIK \\ Department of Chemistry, Jadavpur University, Kolkata-700 032, India \\ mallikak52@yahoo.co.in
}

Received 18 September 2012 / Accepted 20 October 2012

\begin{abstract}
A rapid, efficient and green methodology has been developed for the synthesis of 3,3'-arylmethylene-bis-4-hydroxycoumarins by microwave assisted condensation of aromatic aldehydes and 4-hydroxycoumarin without use of any solvent, catalyst or solid surface.
\end{abstract}

Keywords: 3,3'-Arylmethylene-bis-4-hydroxycoumarins, Solvent-free synthesis, Catalyst-free synthesis, 4-Hydroxycoumarin

\section{Introduction}

4-Hydroxycoumarin and its derivatives are known for their anticoagulant ${ }^{1}$, antibacterial ${ }^{2}$, antifungal $^{2}$, antibiotic ${ }^{3}$, antitumor ${ }^{3,4}$ and anti-HIV ${ }^{5}$ activities. They are also used as agrochemicals $^{6}$ and analytical reagents ${ }^{7}$. 3,3'-Arylmethylene-bis-4-hydroxycoumarins (3), commonly known as biscoumarins, are the bridge substituted dimers of 4-hydroxycoumarin. They have enormous potential as anticoagulants ${ }^{1,8,9}$ and antioxidants ${ }^{6}$ and some of them have also been found to be urease inhibitors ${ }^{10}$. The compound 3,3-methylene-bis-4hydroxycoumarin, commonly known as dicoumarol, occurs naturally in moldy clover ${ }^{11}$. It is the hemorrhagic agent responsible for the sweet clover disease of cattle and has also been employed for the prevention and treatment of thrombosis ${ }^{12}$. 3,3'-Arylmethylene-bis-4hydroxycoumarins (3) are usually synthesized by condensing 4-hydroxycoumarin (1) with various aldehydes (aromatic, heterocyclic and $\alpha, \beta$-unsaturated) (2) using different catalysts and media ${ }^{8-10,13-19}$. Some of these methods require long reaction time, use of expensive catalysts and organic solvents and tedious work up ${ }^{8,10}$. The current literature shows that there has been a growing trend towards green synthesis of these compounds ${ }^{13-19}$. However, in such reported green methods use of catalysts, solid surfaces, solvents etc. could not be avoided. The current trend towards development of catalyst-free and solvent-free reaction conditions for organic synthesis ${ }^{20-23}$ encouraged us to study the same reaction under microwave irradiation condition without using any solvent, catalyst or solid surface. The remarkable success in this endeavor is presented herein. 
2<smiles>O=c1cc(O)c2ccccc2o1</smiles>

1
ArCHO $\underset{\text { MW Irradiation }}{2.5-3.5 \text { min }}$ 2<smiles>O=c1oc2ccccc2c(O)c1C([Al])c1c(O)c2ccccc2oc1=O</smiles>

3

Scheme 1. Synthesis of 3,3'-arylmethylene-bis-4-hydroxycoumarins (3)

\section{Experimental}

Melting points were recorded on a Köfler block. IR spectra were recorded on a Perkin Elmer FT-IR spectrophotometer (Spectrum BX II) in $\mathrm{KBr}$ pellets. ${ }^{1} \mathrm{H}$ and ${ }^{13} \mathrm{C}$ NMR spectra were recorded in $\mathrm{CDCl}_{3}$ on a Bruker AV-300 (300 MHz) spectrometer. Analytical samples were routinely dried in vacuo at room temperature. Microanalytical data were recorded on a Perkin-Elmer 2400 Series II C, H, N analyzer. The FAB-MS of $\mathbf{3 j}$ was recorded on a Jeol the M Station JMS.700 spectrometer. Column chromatography was performed with silica gel (100-200 mesh) and TLC with silica gel G made of SRL Pvt. Ltd. Petroleum ether had the boiling range $60-80{ }^{\circ} \mathrm{C}$.

\section{General procedure for synthesis of 3,3'-arylmethylene-bis-4-hydroxycoumarins (3)}

In a typical experiment, an intimate mixture of 4-hydroxycoumarin (1, 4 mmole) and an aromatic aldehyde (2, 2 mmole) was taken in a pyrex beaker $(100 \mathrm{~mL})$ and it was irradiated with microwave for 2.5-3.5 min within which the reaction was complete (Table 1) [An unmodified domestic household microwave oven (LG, DMO, Model No.-556P, 900 watt) was used. The MW oven was operated at reduced MW-power level of $60 \%$ (540 watt).]. The reaction mixture was then cooled and crystallized from $\mathrm{CH}_{2} \mathrm{Cl}_{2}$ - petroleum ether, which gave $\mathbf{3}$ in perfectly pure state.

All the thirteen compounds of the series 3 synthesized by us have been found to be previously known. The analytical and spectral data of some selected compounds are given below:

\section{3,3'-Phenylmethylene-bis-4-hydroxycoumarin (3a)}

Colourless crystalline solid, IR ( $\left.\mathrm{KBr}, v \mathrm{~cm}^{-1}\right)$ : 3069, 1660 (C=O), 1616, 1568, 1496, 1337, 1266, $1199(\mathrm{OH}), 1093,902,800,757 ;{ }^{1} \mathrm{H}$ NMR (300 MHz, $\left.\mathrm{CDCl}_{3}\right): \delta=6.11$ (s, $1 \mathrm{H}, \mathrm{Ar}-$ $\mathrm{CH}<$ ),7.21-7.43 (m, 9H), 7.63 (dt, 2H, $J=7.9$ and $1.5 \mathrm{~Hz}$ ), 8.01(br. d, $1 \mathrm{H}, J=6.7 \mathrm{~Hz}$ ), 8.07 (br.d, $1 \mathrm{H}, J=6.6 \mathrm{~Hz}$ ), 11.30 (br. s, $1 \mathrm{H}, \mathrm{OH}$ ), 11.53 (br. s, $1 \mathrm{H}, \mathrm{OH}$ ); Anal. Calcd. for $\mathrm{C}_{25} \mathrm{H}_{16} \mathrm{O}_{6}$ C, 72.81; H, 3.91\%; found C, $72.63 ; \mathrm{H}, 4.02 \%$.

\section{3,3'-(4-Methoxyphenylmethylene)-bis-4-hydroxycoumarin (3c)}

Colourless crystalline solid, IR (KBr, $\left.v \mathrm{~cm}^{-1}\right)$ : $3440(\mathrm{OH}), 3072,3002,1668(\mathrm{C}=\mathrm{O}), 1604$, 1565, 1510, 1454, 1353, 1258, $1180(\mathrm{OH}), 1094,907,828,768 ;{ }^{1} \mathrm{H}$ NMR (300 MHz, $\mathrm{CDCl}_{3}$ ): $\delta=3.80$ (s, 3H, $\mathrm{OCH}_{3}$ ), 6.05 (s, $1 \mathrm{H}, \mathrm{Ar}-\mathrm{CH}<$ ), 6.85 ( d, $2 \mathrm{H}, J=8.7 \mathrm{~Hz}$ ), 7.13 (d, $2 \mathrm{H}, J=8.7 \mathrm{~Hz}$ ), $7.30-7.42$ ( m, 4H), 7.63 (br. t, $2 \mathrm{H}, J=8.2 \mathrm{~Hz}$ ), 8.03 (dd, 2H, $J=8.4 \mathrm{~Hz}$ ), 11.29 (br. s, $1 \mathrm{H}, \mathrm{OH}$ ), 11.51 (br. s, $1 \mathrm{H}, \mathrm{OH}$ ) ; Anal. Calcd. for $\mathrm{C}_{26} \mathrm{H}_{18} \mathrm{O}_{7}$ C, 70.58; $\mathrm{H}$, 4.10\%; found C, 70.37; H, 4.22\%. 


\section{3,3'-(4-Chlorophenylmethylene)-bis-4-hydroxycoumarin (3d)}

Colourless crystalline solid, IR (KBr, $\left.v \mathrm{~cm}^{-1}\right)$ :3072, 2684, 2609, 1668 (C=O), 1617, 1603, 1490, 1454, 1351, 1311, 1266, $1182(\mathrm{OH}), 1094,920,908,821,790,706 ;{ }^{1} \mathrm{H}$ NMR (300 $\mathrm{MHz}, \mathrm{CDCl}_{3}$ ): $\delta=6.04$ (s, $1 \mathrm{H}, \mathrm{Ar}-\mathrm{CH}<$ ), 7.15 (d, 2H, $J=8.4 \mathrm{~Hz}$ ), 7.29 (d, $2 \mathrm{H}, J=8.6 \mathrm{~Hz}$ ), 7.38-7.43 (m, 4H), 7.64 (dt, 2H, $J=8.0$ and $1.5 \mathrm{~Hz}$ ), 8.00 (br. D, $1 \mathrm{H}, J=7.3 \mathrm{~Hz}$ ), 8.07 (br. D, $1 \mathrm{H}, J=7.2 \mathrm{~Hz}$ ), 11.31 (br. S, $1 \mathrm{H}, \mathrm{OH}$ ), 11.53 (br. S, 1H, OH); Anal. Calcd. For $\mathrm{C}_{25} \mathrm{H}_{15} \mathrm{ClO}_{6}$; C, 67.20; H, $3.38 \%$; found C, 66.96; H, 3.52\%.

\section{3,3'-(4-Bromophenylmethylene)-bis-4-hydroxycoumarin (3e)}

Colourless crystalline solid, IR $\left(\mathrm{KBr}, v \mathrm{~cm}^{-1}\right)$ : $3446(\mathrm{OH}), 3071,2729,2610,2361,1668$ (C=O), 1618, 1604, 1561, 1488, 1351, 1309, 1266, $1182(\mathrm{OH}), 1094,908,820,766 ;{ }^{1} \mathrm{H}$ NMR (300 MHz, $\left.\mathrm{CDCl}_{3}\right): \delta=6.01(\mathrm{~s}, 1 \mathrm{H}, \mathrm{Ar}-\mathrm{CH}<), 7.10(\mathrm{~d}, 2 \mathrm{H}, J=8.2 \mathrm{~Hz}), 7.32-7.45(\mathrm{~m}$, 6H), 7.64 (dt, 2H, $J=8.0$ and $1.5 \mathrm{~Hz}$ ), 7.99 (br. D, 1H, $J=7.7 \mathrm{~Hz}$ ), 8.06 ( br. D, $1 \mathrm{H}, J=7.8$ $\mathrm{Hz}$ ), 11.31 (br. S, $1 \mathrm{H}, \mathrm{OH}$ ), 11.54(br. S, $1 \mathrm{H}, \mathrm{OH}$ ); Anal. Calcd. For $\mathrm{C}_{25} \mathrm{H}_{15} \mathrm{BrO}_{6}$; C, 61.12; H, 3.08\%; found C, 61.21; H, 3.24\%.

\section{3,3'-(4-Nitrophenylmethylene)-bis-4-hydroxycoumarin (3h)}

Colourless crystalline solid, IR (KBr, $\left.v \mathrm{~cm}^{-1}\right)$ : $3440(\mathrm{OH}), 3072,2361,1660(\mathrm{C}=\mathrm{O}), 1618$, 1601, 1566, 1519, 1494, 1348, 1309, 1265, $1182(\mathrm{OH}), 1109,909,826,765 ;{ }^{1} \mathrm{H}$ NMR (300 $\mathrm{MHz}, \mathrm{CDCl}_{3}$ ): $\delta=6.12$ (s, $1 \mathrm{H}, \mathrm{Ar}-\mathrm{CH}<$ ), 7.40-7.45 (m, 6H), 7.67(br. T, 2H, $J=7.8 \mathrm{~Hz}$ ), 8.01(br. D, $1 \mathrm{H}, J=7.6 \mathrm{~Hz}$ ), 8.11 (br. D, $1 \mathrm{H}, J=7.2 \mathrm{~Hz}$ ), 8.19 (d, $2 \mathrm{H}, J=8.9 \mathrm{~Hz}$ ), 11.37 (s, $1 \mathrm{H}, \mathrm{OH}$ ), 11.57 (s, $1 \mathrm{H}, \mathrm{OH}$ ); Anal. Calcd. For $\mathrm{C}_{25} \mathrm{H}_{15} \mathrm{NO}_{8}$; C, 65.65; H, 3.31; N, 3.06\% found C, 65.38; H, 3.22; N, 3.20\%.

\section{3,3'-(4-Hydroxy-3-methoxyphenylmethylene)-bis-4-hydroxycoumarin (3j)}

Colourless crystalline solid, IR ( $\left.\mathrm{KBr}, v \mathrm{~cm}^{-1}\right)$ : $3457(\mathrm{OH}), 2360,1668(\mathrm{C}=\mathrm{O}), 1616,1604$, 1515, 1451, 1352, 1270, 1212, $1187(\mathrm{OH}), 1093,909,799,766 ;{ }^{1} \mathrm{H}$ NMR (300 MHz, $\mathrm{CDCl}_{3}$ ): $\delta 3.75$ (s, 3H, $\mathrm{OCH}_{3}$ ), 5.58 (br. S, $\left.1 \mathrm{H}, \mathrm{OH}\right), 6.06$ (s, $1 \mathrm{H}, \mathrm{Ar}-\mathrm{CH}<$ ), $6.67(1 \mathrm{H}, \mathrm{s})$, 6.72 (d, 1H, $J=8.4 \mathrm{~Hz}$ ), 6.86 (d, 1H, $J=8.3 \mathrm{~Hz}$ ), 7.36-7.42 (m, 4H), 7.63 (br. T, 2H, $J=7.6$ Hz), 8.03 ( br. Peak, 2H, w $w_{1 / 2}=23.9 \mathrm{~Hz}$ ), 11.28 (br. S, $1 \mathrm{H}, \mathrm{OH}$ ), 11.51 (br. S, $1 \mathrm{H}, \mathrm{OH}$ ); ${ }^{13} \mathrm{C}$ NMR (300 MHz, $\left.\mathrm{CDCl}_{3}\right) \delta 146.70,144.56,132.83,126.85,124.89,124.36,119.51,116.64$, 114.46, 109.44, $56.10\left(\mathrm{OCH}_{3}\right), 35.79(\mathrm{Ar}-\mathrm{CH}<)$; FABMS: $458.3\left(\mathrm{M}^{+}\right)$; Anal. Calcd. For $\mathrm{C}_{26} \mathrm{H}_{18} \mathrm{O}_{8}$; C, 68.12; H, 3.96\%; found C, 67.95; H, 4.11\%.

\section{3,3'-(2- Thiophenyl)-bis-4-hydroxycoumarin (3l)}

Greenish crystalline solid, IR (KBr, $\left.v \mathrm{~cm}^{-1}\right)$ : 3069, 1655, 1616, 1602, 1568, 1358, 1309, 1268, 1098, 903, 810, 761, 720, 707. ${ }^{1} \mathrm{H}$ NMR (500 MHz, $\left.\mathrm{CDCl}_{3}\right): \delta 6.20$ (s, 1H), 6.85-6.86 (m, 1H), 6.94-6.96 (m, 1H), 7.21-7.22 (m, 1H), 7.40-7.41 (m, 4H), 7.61-7.65 (m, 2H), 8.04 (br. S, $2 \mathrm{H}, \mathrm{w}_{1 / 2}=32 \mathrm{~Hz}$ ), 11.27 (s, $\left.1 \mathrm{H}, \mathrm{OH}\right), 11.79$ (s, $1 \mathrm{H}, \mathrm{OH}$ ); Anal. Calcd. For $\mathrm{C}_{23} \mathrm{H}_{14} \mathrm{O}_{6} \mathrm{~S}$; C, 66.02; H, 3.37\%; found C, 66.29; H, 3.58\%.

\section{Results and Discussion}

Our present method involves subjecting of a mixture of an aldehyde and 4-hydroxycoumarin in 1:2 mole ratio directly to microwave irradiation. A range of structurally diverse aldehydes belonging to the categories aromatic, heterocyclic and $\alpha, \beta$-unsaturated aldehydes were taken. To our delight, the target compounds were obtained in excellent yield in the above mentioned method for all the combinations. The yields of the products by the method have been presented in Table 1 . 
Table 1. Synthesis of 3,3'-Arylmethylene-bis-4-hydroxycoumarins (3) from 4-hydroxycoumarin (1) by MW Irradiation

\begin{tabular}{|c|c|c|c|c|c|}
\hline Entry & $\begin{array}{l}\text { Aldehyde } \\
\text { (2) }\end{array}$ & $\mathrm{Ar}$ & $\begin{array}{l}\text { Time, } \\
\text { min }\end{array}$ & $\begin{array}{l}\text { Yield of 3, } \\
\%^{\dagger}[3 \mathbf{a}-\mathbf{m}]\end{array}$ & $\mathrm{mp},{ }^{\circ} \mathrm{C}$ [Lit. Value] \\
\hline 1 & $2 a$ & $\mathrm{C}_{6} \mathrm{H}_{5-}^{-}$ & 3.5 & 91 & $231-232[230-232]^{13}$ \\
\hline 2 & $2 \mathbf{b}$ & 4-Me- $\mathrm{C}_{6} \mathrm{H}_{4}-$ & 3.5 & 93 & $265-266[266-268]^{13}$ \\
\hline 3 & $2 c$ & $4-\mathrm{MeO}-\mathrm{C}_{6} \mathrm{H}_{4-}^{-}$ & 3.5 & 95 & $244-245[246-248]^{13}$ \\
\hline 4 & 2d & $4-\mathrm{Cl}-\mathrm{C}_{6} \mathrm{H}_{4^{-}}$ & 2.5 & 95 & $257-258[256-258]^{13}$ \\
\hline 5 & $2 e$ & $4-\mathrm{Br}-\mathrm{C}_{6} \mathrm{H}_{4}^{-}$ & 3.5 & 92 & $268-270[265-267]^{13}$ \\
\hline 6 & $2 f$ & $4-\mathrm{Me}_{2} \mathrm{~N}-\mathrm{C}_{6} \mathrm{H}_{4^{-}}$ & 3.5 & 91 & $225-227[222-224]^{13}$ \\
\hline 7 & $2 g$ & $3-\mathrm{O}_{2} \mathrm{~N}-\mathrm{C}_{6} \mathrm{H}_{4^{-}}$ & 3.5 & 94 & $233-234[234-236]^{13}$ \\
\hline 8 & $2 \mathrm{~h}$ & $4-\mathrm{O}_{2} \mathrm{~N}-\mathrm{C}_{6} \mathrm{H}_{4}^{-}$ & 3.5 & 97 & $234-235[232-234]^{13}$ \\
\hline 9 & $2 \mathbf{i}$ & & 3.5 & 93 & $258-259[260]^{13}$ \\
\hline 10 & $2 \mathbf{j}$ & $\mathrm{MeO}^{\prime}$ & 3.5 & 92 & $227-228^{\pi}$ \\
\hline 11 & $2 \mathbf{k}$ & 2-Furyl & 2.5 & 94 & $204-205[202]^{13}$ \\
\hline 12 & 21 & 2-Thienyl & 3.5 & 93 & $213-214[210]^{13}$ \\
\hline 13 & $2 \mathrm{~m}$ & $E-\mathrm{C}_{6} \mathrm{H}_{5} \mathrm{CH}=\mathrm{CH}-$ & 3.5 & 89 & $232-233[230-232]^{13}$ \\
\hline
\end{tabular}

${ }^{\dagger}$ Pure product obtained after crystallization. "The compound has been reported in ref. 25 , but its $m p$ has not been given there.

In the method being reported it was a common observation that the reactions were very clean and no side product was formed in any run. In fact, the crude products obtained were of high purity ( $>95 \%$ by ${ }^{1} \mathrm{H} \mathrm{NMR}$ ) and did not require any chromatographic separation. Their crystallization from $\mathrm{CH}_{2} \mathrm{Cl}_{2}$ - petroleum ether provided analytically pure samples. More significantly, the whole operation did not require any solvent, organic or inorganic, at any stage. Furthermore, the reaction condition has been found to be mild enough to tolerate a variety of functionalities such as $\mathrm{NO}_{2}, \mathrm{Cl}, \mathrm{OH}, \mathrm{OMe}$, conjugated $\mathrm{C}-\mathrm{C}$ double bond and heterocyclic moieties. It is very likely that in our experiments 4-hydroxycoumarin itself or a carboxylic acid formed by aerial oxidation of the aldehyde used acts as acid catalyst and so addition of any external catalyst does not become necessary.

\section{Conclusion}

We have developed a very simple, efficient and environmentally benign method for synthesis of 3,3'-arylmethylene-bis-4-hydroxycoumarins (3) without use of any catalyst, solvent, surfactant or solid support. We feel that this protocol being a good addition to the currently reported methods ${ }^{24,25}$ is in fact better than many others referred herein.

\section{Acknowledgement}

Financial assistance from the UGC-CAS and DST-PURSE programs, Department of Chemistry is gratefully acknowledged. The authors also acknowledge the DST-FIST program to the 
Department of Chemistry, Jadavpur University for providing the NMR spectral data. AD and SS are thankful to the CSIR and RM to the UGC, New Delhi for their Research Fellowships.

\section{References}

1. Overmann R S, Stahmann M A, Heubner C F, Sullivan W R, Spero L, Doherty D G, Ikawa M, Graf L, Roseman S and Lonk K P, J Biol Chem., 1944, 153(1), 5-24.

2. Chohan Z H, Shaikh A U, Rauf A and Supuran C T, J Enzym Inhib Med Chem., 2006, 21(6), 741-748.

3. Jung J, Lee J H, Oh S and Lee J G, Bioorg Med Chem Lett., 2004, 14, 5527-5531.

4. Chen Y L, Wang T C, Tzeng C C and Chang N C, Helv Chim Acta, 1999, 82, 191-197.

5. Zhao H, Neamati N, Hong H, Mazumder A, Wang S, Sunder S, Milne G W A, Pommier Y and Burke T R, J Med Chem., 1997, 40(2), 242-249.

6. Kancheva V D, Boranova P V, Nechev J T and Monolov I I, Biochimie, 2010, 92, 1138-1146.

7. Bhat A N and Jain B D, Talanta, 1960, 5(3-4), 271-275.

8. Appendino G, Cravotto G, Tagliapietra S, Ferraro S, Nano G M and Palmisano G, Helv Chim Acta, 1991, 74(7), 1451-1458.

9. Manolov I, Moessmer C M and Danchev N, Eur J Med Chem., 2006, 41(7), 882-890.

10. Khan K M, Iqbal S, Lodhi M A, Maharvi G M, Zia-u-Allah, Choudhary M I, Rahman A U and Perveen S, Bioorg Med Chem., 2004, 12, 1963-1968.

11. Murray R D H, Mendez J and Brown S A, The Natural Coumarins; Wiley: Chichester, 1982.

12. Lehmann J, The Lancet, 1943, 241, 611-613.

13. Kidwai M, Bansal V, Mothsra P, Saxena S, Somvanshi R K, Dey S and Singh T P, $J$ Mol Catal A Chem., 2007, 268, 76-81.

14. Khurana J M and Kumar S, Tetrahedron Lett., 2009, 50(28), 4125-4127.

15. Mehrabi H and Abusaidi H, J Iran Chem Soc., 2010, 7(4), 890-894.

16. Qadir S, Dar A A and Khan K Z, Synth Commun., 2008, 38, 3490-3499.

17. Zhou J-F, Gong G- X, An L-T, Sun X-J and Zhu F-X, Chin J Org Chem., 2009, 29(12), 1988-1991.

18. Završnik D, Muratović S, Makuc D, Plavec J, Cetina M, Nagl A, Clercq E D, Balzarini J and Mintas M, Molecules, 2011, 16(7), 6023-6040.

19. Davoodnia A, Bull Korean Chem Soc., 2011, 32, 4286-4290.

20. Cozzi P G and Zoli L, Green Chem., 2007, 9(12), 1292-1295.

21. Cozzi P G and Zoli L, Angew Chem Int Ed., 2008, 47, 4162-4166.

22. Mashkouri S and Naimi-Jamal M R, Molecules, 2009, 14(1), 474-479.

23. Galleti P, Pori M and Giacomini D, Eur J Org Chem., 2011, 2011(20-21), 3896-3903.

24. Tabatabaeian K, Heidari H, Khorshidi A, Mamaghani M and Mahmoodi N, J Serb Chem Soc., 2012, 77(4), 407-413.

25. Karmakar B, Nayak A and Banerji J, Tetrahedron Lett., 2012, 53(33), 4343-4346. 